\title{
Laser beam zooming and deflection using a nonlinear metamaterial refracting medium
}

\author{
Andrew James Comley* \\ AWE Aldermaston, Reading RG74PR, Berkshire, UK
}

Received: 21 April 2017 / Accepted: 31 January 2018

\begin{abstract}
In-process control of the focal spot size and pointing position of a laser as it interacts with a target (beam zooming and deflection) offers the possibility of unprecedented efficiency improvements in a number of applications, such as inertial confinement fusion and laser micromachining. Here is described a system in which the focussing characteristics of a laser beam at one wavelength can be controlled by a lower-intensity beam at another wavelength, via their mutual interaction with a nonlinear metamaterial refracting medium. Such a metamaterial approach permits the optical response of the medium to be tailored according to the wavelengths of interest and time response required in a given application. A metamolecule unit cell design is described in terms of an equivalent circuit based on a pair of LCR (inductance, capacitance, resistance) circuits coupled by a common nonlinear capacitor. The circuit is studied using an analytical approach to obtain an understanding of its properties and design relationships between circuit parameters. Potential realisations of the circuit are discussed.
\end{abstract}

Keywords: Laser / Zooming / Deflection / Metamaterial / Nonlinear

\section{Introduction}

The ability to rapidly control the focussing characteristics of a laser as it interacts with a target is potentially advantageous in a number of applications, including laserdriven inertial confinement fusion (ICF) [1-6], laser micromachining [7] and high-pressure materials experiments [8]. In-process control of the laser focal spot size and pointing position (beam zooming and deflection, respectively) offers the possibility of optimising the transfer of energy from the laser to the target at all times, thereby enhancing the desired process without increasing the required laser energy budget.

For example, in direct-drive ICF multiple laser beams heat and compress a capsule containing cryogenic deuterium-tritium fuel from all directions with the aim of initiating fusion burn and energy gain [1-3]. Beam zooming during the capsule implosion has been predicted to result in large efficiency gains [5,6], and ideally zooming would be accomplished on a sub-nanosecond timescale to track the capsule as it implodes. In the polar direct-drive variation of ICF [3], the capsule is driven by two opposed cones of laser beams, one at each pole, such that the illumination intensity over the capsule surface is not uniform. In this case, the ability to both zoom and deflect

\footnotetext{
* e-mail: andrew.comley@awe.co.uk
}

the laser beams during the compression of the capsule may prove beneficial. In indirect-drive ICF [4], laser beams heat the inside of typically a gold, cylindrical enclosure known as a hohlraum. X-rays from the hot gold wall irradiate and ablate the surface of the capsule, thereby driving the implosion of the nuclear fuel. Rapid beam zooming and deflection of the laser beams would allow wall heating and the resulting x-ray field experienced by the capsule to be optimised at all times.

Similarly, in-process beam zooming and deflection could be exploited in laser micromachining applications to precisely control the deposition of laser energy into the workpiece.

Given these anticipated benefits, efforts have already been made to add beam zooming capability to existing laser systems. For example, zooming technology has been demonstrated in excimer laser systems, notably at the NIKE KrF laser facility at the Naval Research Laboratory (NRL) [9]. In order to implement zooming, several parallel beam paths are constructed at the front end (i.e. lowenergy section) of the laser system. Each beamline is nominally identical optically apart from a beam aperture which is imaged onto the target at a given time. By choosing the size of the aperture appropriately in each beamline, a multi-state beam zooming effect can be accomplished. Electro-optical components are used to provide independent control of pulse shape and time of 
arrival for each beamline. By its nature, this architecture permits only a discrete number of zoom states (one for each low-energy beamline) to be implemented.

For solid-state lasers, a two-state zooming optic called the zooming phase plate (ZPP) has been proposed $[5,6]$. The ZPP in effect divides the beam into two distinct regions each of which is focussed independently. It has a central area which produces a larger, low-order Gaussian focal spot for the early stage of an ICF implosion, and an outer area which produces a smaller, high-order Gaussian focal spot for the later stages of the implosion.

Another approach utilises the configuration flexibility of multiple-beam laser facilities such as the National Ignition Facility [10], Omega [11], the Megajoule Laser Facility [12] and Orion [13]. Although the focussing condition of each beam is fixed, beams can be individually timed and focussed to optimise the laser-target energy coupling efficiency as a function of time (i.e. beams fired early in time are chosen to have a larger focal spot than beams fired later in time) $[14,15]$.

Whilst these zooming schemes allow improvements in laser-target energy coupling efficiency to be made, continuous (non-discrete) zooming, if it could be achieved and combined with dynamic beam deflection, would lead to even greater enhancements.

One possible way to effect continuous zooming is to use an electro-optic (EO) crystal to modify the wavefront curvature of the beam and therefore its focussing position relative to the target [16]. However, EO crystals generally have a small beam aperture (e.g. crystal diameter in Ref. 16 is $20 \mathrm{~mm}$ ). This makes them suitable for use only in the early stages of a laser beamline before the beam has been expanded and injected into the amplifier chain. Consequently, the effect of the EO crystal on the performance of later sub-systems, such as spatial filters and frequency conversion crystals, has to be carefully considered.

Alternatively, the wavelength-dependent focussing characteristics of a grating placed at the end of the laser beamline can be exploited to achieve a zooming effect [17]. In this case, the zooming control information is encoded in the time-dependent modulation of the laser pulse frequency, early in the system. The laser pulse has an increased bandwidth as a result of the frequency modulation, and this has an impact on the performance of the beamline. Whilst the effect of increased bandwidth can be offset by increasing the energy injected into the main amplifier, this has implications for staying below damage thresholds for components later in the system.

An alternative approach is to employ a separate control laser to induce refractive index gradients in a medium which then influence the beam to be controlled. Through appropriate choice of the spatiotemporal intensity characteristics of the control beam, a dynamic lens or prism can be 'written' into the refracting medium to effect zooming or deflection of the beam to be controlled, respectively. In general, the controlled beam might be both zoomed and deflected by simultaneously shifting its focus parallel and transverse to the optical axis.

For instance, a control laser beam may be spatially and temporally overlapped with the beam to be controlled in a nonlinear medium, with refractive index variations induced via the optical Kerr effect [18]. It is notable though that in this case the control beam must necessarily be much more intense than the controlled beam to ensure that its contribution to the induced refractive index is dominant.

In an alternative scheme, an atomic gas is employed as the refracting medium [19]. The frequency of the control beam is different to that of the controlled beam but both frequencies are close to characteristic resonance frequencies of the atomic gas. Coupling between resonances is exploited such that the control beam may be made weak (low intensity) compared to the controlled beam. This scheme is therefore reliant on the availability of a suitable gaseous medium with the desired resonance frequencies. In one example [20], an alkali metal gas is employed which requires several auxiliary components such as a gaseous cell, oven and a means of controlling temperature and pressure. The characteristic response time is limited to around a nanosecond.

Given these limitations, it would be preferable to replace atoms in this scheme with 'effective atoms': userdefined resonant structures capable of mimicking the behaviour of atomic resonances in a controllable way, and reacting on a sub-nanosecond timescale. Such a possibility is offered by metamaterials: artificial materials consisting of sub-wavelength, resonant unit cells engineered to achieve a desired effective bulk medium response to electromagnetic radiation [21].

In this paper, a dynamic zooming and deflection system is described which is enabled by a nonlinear, metamaterialbased refracting medium. It is motivated by the desire to use a low-intensity laser to control a high-intensity laser via the refracting medium, but without the limitations of the atomic gas just described. Furthermore, the system is intended to control large-scale fusion or machining lasers on a sub-nanosecond timescale without imposing significant changes on existing facilities.

In Section 2, the overall system is described in general terms and its key features are identified. In Section 3, the proposed metamaterial unit cell is modelled using an equivalent circuit approach and its performance analysed for the case of magnetic field excitation. Possible realisations of the circuit are then described. Finally, the article is summarised in Section 4.

\section{Dynamic zooming and deflection system}

In this section, a system is described in which an optic with time-varying refracting properties, for example a lens or prism, is combined with a static focussing optic to accomplish rapid control of the focussing properties of a laser beam. The dynamic optic is constructed using a metamaterial approach such that its refractive properties are determined by the spatio-temporal intensity characteristics of a control beam, as discussed in Section 3.

In Figure 1, the dynamic optic is shown inserted near the end of an existing laser beamline. It might be placed just before or after the final focussing optic, or as a metamaterial layer coated on its surface. 


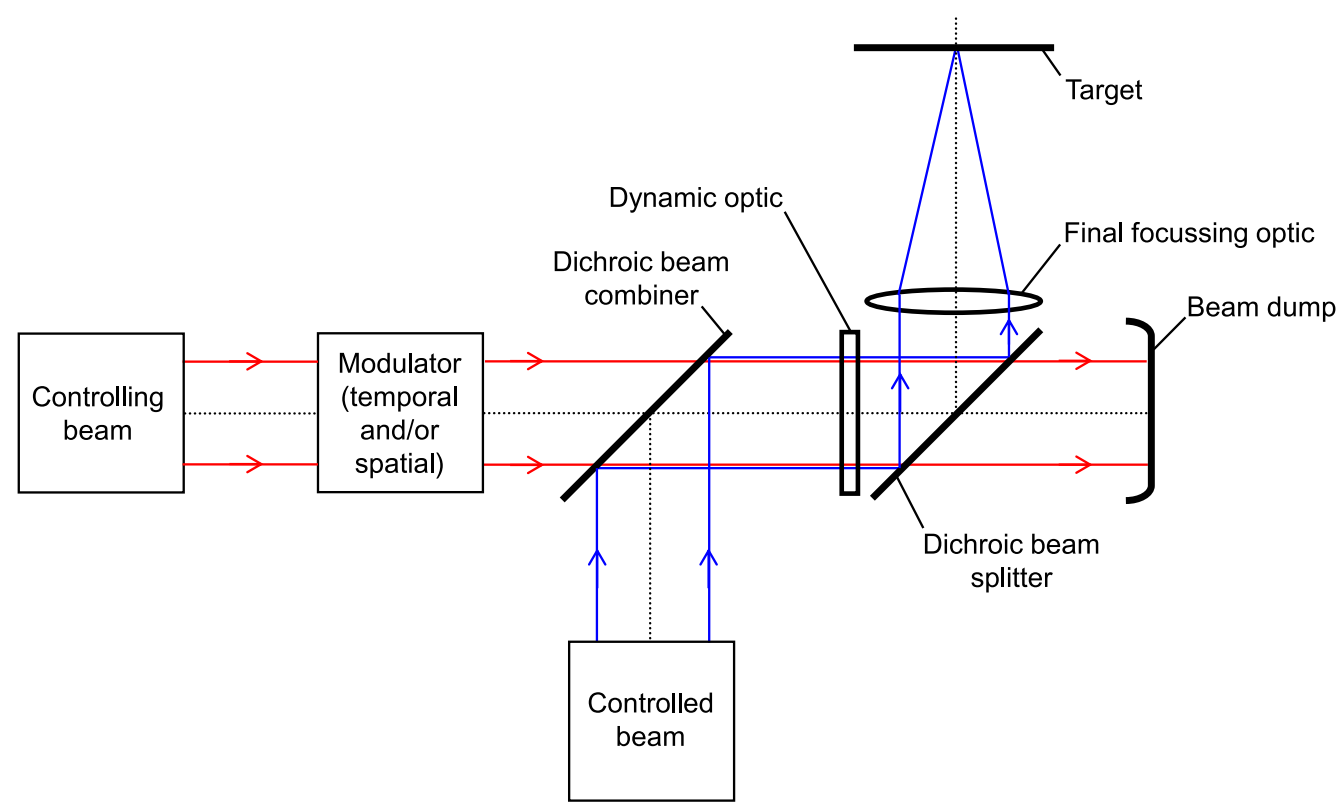

Fig. 1. Two-colour zooming and deflection system.

The control and controlled beams, which are assumed to have different wavelengths, are combined and later separated using dichroic optics. However, the dichroic optics could be eliminated by using existing components to perform their functions. The function of the combining optic may be performed by the final turning mirror, if it has suitable reflection and transmission characteristics. For example, the mirror might employ a wavelength-selective coating to provide high reflectivity at the controlled beam wavelength, which may also be highly transmitting at the control beam wavelength. The function of the dichroic beamsplitter might be performed by the final focussing optic, by exploiting chromatic aberration to defocus and separate the control beam relative to the controlled beam on target [22].

Note that as the dynamic optic is positioned near the end of the beamline, the controlled beam propagation characteristics up to the final focussing lens do not need to be modified in order to accommodate it i.e. its impact on the existing system is minimal. Furthermore, as the properties of the dynamic optic are set at-a-distance by the control beam, no power supply or other auxiliary components are required in its vicinity. As discussed in the next section, the metamaterial nature of the refracting medium ensures that its response to the desired laser operating frequencies, and its characteristic response timescale, can be tuned as required through appropriate design of the unit cell structure and choice of constituent materials. In practice, the response timescale of the overall system may be limited by the rate at which the intensity of the control beam can be varied. For example, current electro-optic pulse modulation technology permits temporal shaping with $\sim 100 \mathrm{ps}$ resolution [23]. When the controlled beam is operated in multi-pulse mode, the dynamic optic can be varied between pulses as well as during each pulse. The maximum aperture size of the controlled beam is set by the limits of the metamaterial construction.

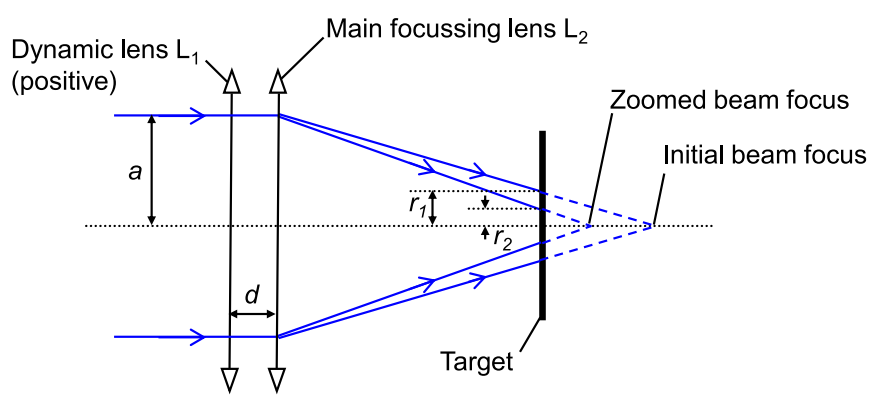

Fig. 2. Simplified ray diagram for zooming system using a dynamic positive lens. The controlling beam is not shown for clarity.

It is desirable that the peak intensity requirement of the control beam be made as small as possible, to minimise the cost of the control beam system. However, a simple thin lens analysis suggests that even a weak dynamic lens could provide a useful zooming effect.

Using for example a control beam with a parabolic spatial intensity distribution, and assuming a linear relationship between the control beam intensity and refractive index change experienced by the controlled beam, a lens can be written into the metamaterial refracting medium (Appendix A). The additional dynamic lens has the effect of shifting the controlled beam focus along the optical axis, thereby producing a zooming effect on target. Taking the radius of the spot size of the controlled beam on target without and with the dynamic lens to be $r_{1}$ and $r_{2}$, respectively, and defining the zooming factor $Z=r_{2} / r_{1}$, a simple thin lens analysis (Fig. 2) yields

$$
Z=\frac{r_{2}}{r_{1}} \sim 1+\frac{f_{2}}{f_{1}}\left(1-\frac{a}{r_{1}}\right),
$$


where $f_{1}$ and $f_{2}$ are the focal lengths of the dynamic lens and main focussing lens respectively, and $a$ is the radius of the controlled beam prior to focussing. This result assumes the lens separation $\mathrm{d}<<f_{1}$. As $a / r_{1}>>1$ and taking $Z$ to be on the order of (but less than) unity, it follows that $f_{1}>>f_{2}$ i.e. that the dynamic lens may be made far weaker than the main focussing lens. A similar result holds when the dynamic lens is negative. The power requirements of the control beam can be reduced by dividing the controlled beam aperture into several Fresnel zones [Appendix A]. In addition to controlling focussed beams, the dynamic lens can in principle be used to vary the divergence of nominally collimated beams.

\section{Metamaterial unit cell}

\subsection{Equivalent circuit}

Having considered the overall beam zooming and deflection system in the previous section, attention is now turned to how the dynamic optic might be realised: it is composed of a nonlinear refracting medium with its desired properties being achieved using a metamaterial approach. The medium is built up from an array of sub-wavelength unit cells, each of which can be described by an equivalent lumped-element circuit. Before considering the circuit in detail, related circuits previously described in the literature are first discussed.

The simplest resonant lumped-element circuit is a single LCR (inductance, capacitance, resistance) circuit, as shown in Figure 3a. Its resonance frequency $\omega_{0}=(L C)^{-0.5}$ and resonance width $\Delta \omega_{0} \sim R / L$ are determined by its lumped circuit parameters which are in turn dependent on the structure and material composition of the unit cell. A well-known realisation of the basic LCR circuit is the splitring resonator (SRR) shown illustratively in Figure 3b. In this case the lumped circuit parameters are given by the approximate relations [24] $L \sim \mu_{0} l^{2} / t, C \sim \varepsilon_{0} w t / d_{g}$ and $R \sim 4(l-w) \rho / w t$, where $\varepsilon_{0}$ and $\mu_{0}$ are the permittivity and permeability of free space, respectively, and $\rho$ is the resistivity of the ring material.

An electromagnetic wave may couple with this circuit either via its magnetic field inducing a voltage and current around the loop via Faraday's Law, or via its electric field applied directly across the capacitance gap [25]. In either case, the field must be orientated appropriately in relation to the circuit loop to produce a resonant response. The induced current flowing around the loop produces a magnetic moment, and by extension a material composed of an array of such SRR's will exhibit a relative permeability and refractive index different to unity.

Whilst considerable flexibility is offered even by this simple unit cell at the design stage, it is naturally desirable to develop structures with properties which can be modified dynamically during operation. This can be accomplished by introducing nonlinear elements into the basic SRR circuit, for example by connecting a varactor diode across the capacitance gap. In this case the capacitance of the varactor diode, and therefore the (a)

(b)
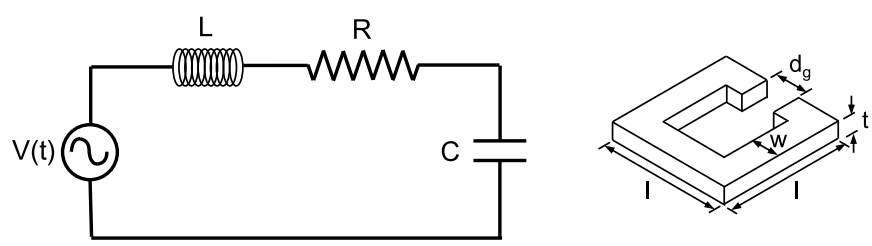

Fig. 3. (a) LCR meta-atom equivalent circuit (b) split-ring resonator unit cell.

resonance frequency of the circuit, is dependent on the power of the exciting electromagnetic wave and the voltage bias across the diode [26-28].

The circuit response can be further tuned by combining the SRR and varactor with other lumped elements. An inductor for instance, when added in parallel with the varactor, can change the sign of the resonance frequency shift [29].

Semiconductor elements can also be incorporated into the basic SRR circuit, and controlled by bias voltages $[30,31]$ or external light sources. In the latter case, a photodiode connected in parallel with the varactor can provide a bias voltage dependent on the intensity of the illuminating light source $[32,33]$.

Alternatively, by creating photoelectrons in a silicon region incorporated into the SRR circuit, its conductivity and therefore the total reactance in the circuit can be controlled [34,35]. In this case the control wavelength must necessarily be shorter than the controlled wavelength, the two wavelengths corresponding to photon energies above and below the semiconductor bandgap energy, respectively. As described in the next section, the opposite is true for the present case: the control wavelength is longer than the controlled wavelength. This feature is particularly advantageous when the controlled electromagnetic radiation has a wavelength in the visible range of the spectrum. In this case, sources of longer-wavelength (e.g. infra-red) control electromagnetic radiation are readily available. Shorterwavelength (e.g. ultraviolet) sources, though available, are often derived from these longer-wavelength sources by frequency conversion methods in nonlinear crystals [36]. Frequency conversion comes at the expense of increased complexity and energy losses incurred in the conversion process. It is therefore preferable to control visible electromagnetic radiation sources with longer-wavelength sources.

As anticipated by Pendry [37], the local electric field in the capacitance gap of a SRR can be significantly higher than the electric field in vacuum. This creates the possibility of strongly enhanced nonlinear effects, for example by inserting a Kerr dielectric medium into the gap [38]. In this case the capacitance takes the form $C_{N L}=\varepsilon_{N L} w t / d_{g}$ with the nonlinear permittivity $\varepsilon_{N L} \sim \varepsilon_{0}\left(1+\chi^{(1)}+\chi^{(3)} E_{g}^{2}\right)$, where $\chi^{(1)}$ and $\chi^{(3)}$ are the first and third order susceptibilities, respectively, and $E_{g}$ is the electric field in the capacitor gap. This can be written in the alternative form used later $C_{N L}=C_{0}+\eta E_{g}^{2}$, where $C_{0}=\varepsilon_{0}\left(1+\chi^{(1)}\right) w t / d_{g}$ and $\eta=\varepsilon_{0} \chi^{(3)} w t / d_{g}$. The nonlinear 


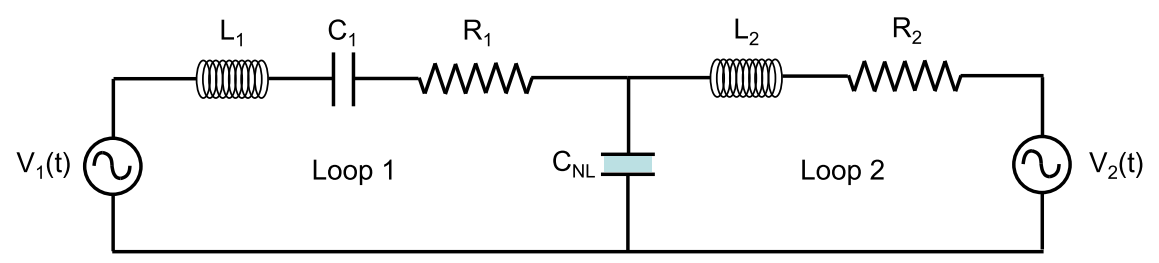

Fig. 4. Dual-resonant equivalent circuit.

capacitance, and therefore the resonance frequency of the circuit, is dependent on the power of the exciting electromagnetic wave.

In this paper, these concepts are extended to a dualresonant system consisting of two LCR circuits directly coupled by a common nonlinear capacitor (Fig. 4). This system is analogous to the resonant gas system proposed in Ref. [19], being designed to act as an intermediary between a control beam and a controlled beam. As the control signal is optical rather than electrical, biasing circuitry to individual pixels required in some schemes [30] is not required. This approach therefore simplifies the design of the metamaterial, especially when structures are scaled down for operation at shorter wavelengths.

As discussed in the next section, this circuit can be realised using a pair of SRR's when operating in the appropriate wavelength regime. Other dual-SRR structures, utilising indirect rather than direct coupling, have been described previously. For example, Slobozhanyuk et al. [39] described a system in which one SRR containing a light-emitting diode (LED) controls a second SRR equipped with a photodiode. The photodiode is arranged to receive the LED signal and sets the bias voltage across a varactor. In another study, Kapitanova et al. demonstrated dual-frequency resonance in a system of two magnetically-coupled SRR's arranged in two spatially-separated and parallel planes [40]. Pendry described pairs of capacitively-coupled SRR's [37], in which one SRR is nested inside the other. These structures can be described by a singly-resonant LCR circuit [41] rather than the dualresonant circuit shown in Figure 4.

Direct (rather than indirect) coupling between a pair of SRR's via a shared component was presented by Filonov et al. [42], similar to the approach taken in the current paper, but with some notable differences. Apart from a difference in the equivalent circuit (Loop 2 in Fig. 4 does not contain a linear capacitor, in contrast to the loop labelled Ring 2 in Fig. 1 of Ref. [42]), the structure of Filonov et al. was designed to operate at resonance at two frequencies for efficient harmonic generation. In the present case, the circuit is designed to operate at resonance at the control wavelength, but slightly off-resonance at the controlled wavelength. These features are described in detail in the next section.

\subsection{Circuit operation}

In this section, the circuit's response to magnetic field (Bfield) excitation is examined using an analytical approach similar to that presented in Ref. [43]. Simple, approximate design equations are derived which relate system performance to the circuit parameters.
The B-field orientations of the control and controlled beams at frequencies $\omega_{1}$ and $\omega_{2}$ respectively are initially chosen such that both beams interact with both circuit loops shown in Figure 4. This results in four voltage sources in the metamolecule circuit as shown in Figure 5a. Voltage $V_{\mathrm{kl}}$ denotes the voltage induced around loop $k$ by beam $l$.

The metamolecule circuit has two resonances, at frequencies $\omega_{01}$ and $\omega_{02}$. In the first resonance mode driven by $V_{11}$ and $V_{21}$, the currents in loop 1 and loop 2 oscillate in the same rotational sense shown by the rotational arrows. In the second resonance mode driven by $V_{22}$ and $V_{12}$, the currents in loop 1 and loop 2 oscillate in the opposite rotational sense with respect to one another.

The first resonance frequency $\omega_{01}$ is set equal to the frequency $\omega_{1}$ of the weak (low-intensity) control beam. This ensures a strong, resonant response such that the voltage across the nonlinear coupling capacitor $C_{\mathrm{NL}}$, and therefore the value of $C_{\mathrm{NL}}$, is dominated by the voltage sources $V_{11}$ and $V_{21}$ associated with the control beam at frequency $\omega_{1}$.

The circuit parameters are chosen as described below such that $\omega_{01}$ is only weakly-dependent on $C_{\mathrm{NL}}$; this ensures that the peak of the resonance curve shifts only slightly relative to $\omega_{1}$ when the control beam turns on. As the peak of the resonance (where the resonance curve is locally flat) is chosen to coincide with $\omega_{1}$ (i.e. $\omega_{01}=\omega_{1}$ ), and by choosing the resistive elements to produce a broad resonance around $\omega_{01}$ (recalling that the resonance width scales linearly with resistance), the change in metamolecule response at $\omega_{1}$ and therefore the refractive index at $\omega_{1}$ due to the shift in the resonance curve is small. This ensures that the effect of the controlled beam on itself via the medium is negligible, and that it is not itself deflected or focussed as it propagates through the refraction medium.

The second resonance frequency of the circuit $\omega_{02}$ is set near (slightly above or below) the frequency $\omega_{2}$ of the strong (high-intensity) controlled beam. Providing the intensity of the controlled beam is not too high, this offresonance condition ensures that the effect of $V_{22}$ and $V_{12}$ on $C_{\mathrm{NL}}$ is less than that of $V_{11}$ and $V_{21}$ (i.e. that the control beam dictates the voltage across the coupling capacitor). The circuit parameters are chosen such that $\omega_{02}$ is stronglydependent on $C_{\mathrm{NL}}$; this ensures that the peak of the resonance curve near $\omega_{2}$ shifts significantly when the control beam turns on. As $\omega_{02}$ is chosen such that $\omega_{2}$ is on the rising edge (or falling edge) of the resonance curve (where the curve is not flat), and by choosing the resistive elements to produce a narrow resonance around $\omega_{02}$, the change in metamolecule response at $\omega_{2}$ and therefore the change in refractive index at $\omega_{2}$ due to the shift in the resonance curve is large. 
(a)
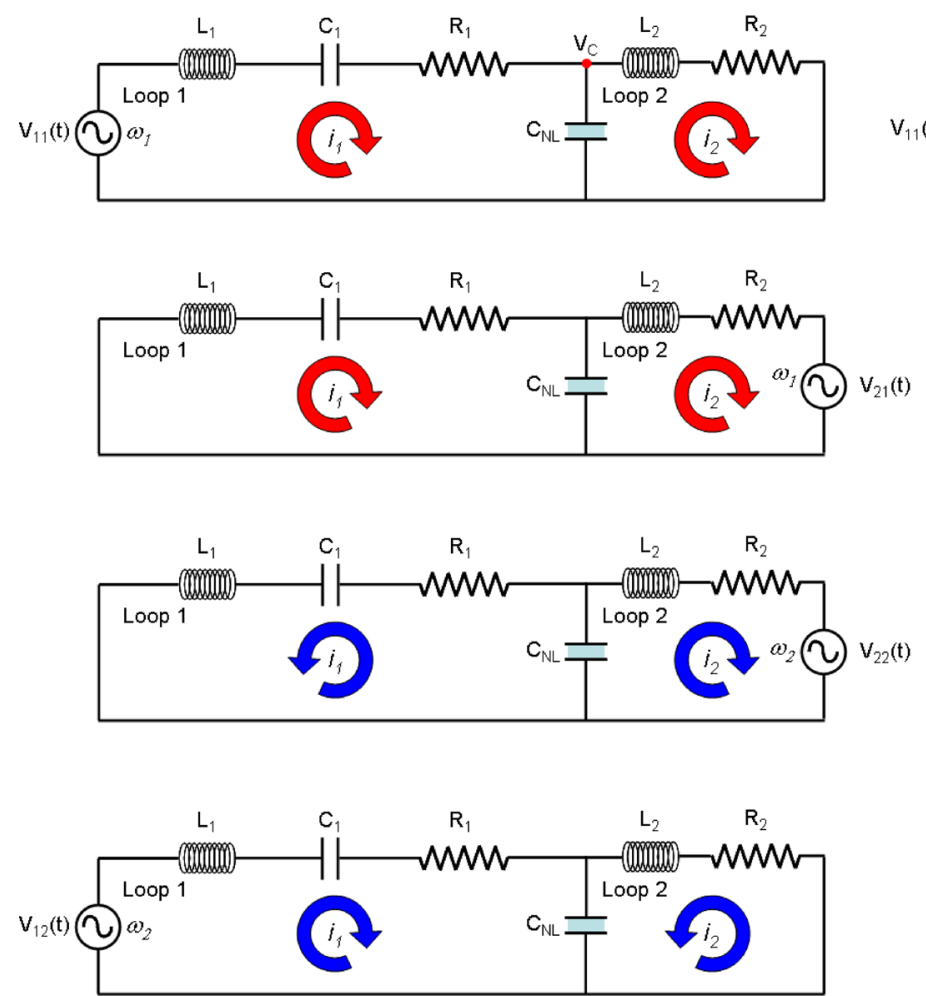

Fig. 5. (a) Dual-resonant circuit seen by each voltage source condition: $\omega_{2}>\omega_{1}, L_{1} \gg L_{2} \rightarrow C_{1} / C_{N L} \gg L_{2} / L_{1}$.

In order to obtain the desired circuit behaviour, the circuit parameters must be chosen accordingly. In the following discussion, the time dependence of the B-field is assumed to vary as $e^{-j \omega t}$; using this convention the complex impedance of an inductor and capacitor is $-j \omega L$ and $j / \omega C$, respectively.

To ensure that the control beam is insensitive to changes in $C_{N L}=C_{0}+\eta E^{2}\left(\eta E^{2}<<C_{0}\right.$, i.e. $\left.C_{N L} \approx C_{0}\right)$, choose

$$
\frac{1}{\omega_{1} C_{0}} \gg \omega_{1} L_{2} \Rightarrow \frac{1}{L_{2} C_{0}} \gg \omega_{1}^{2}
$$

This ensures that the magnitude of the reactance of $C_{\mathrm{NL}}$ is much larger than that of $L_{2}$ at the frequency of the controlling beam $\omega_{1}$. Demanding that the system is resonant at frequency $\omega_{1}$ when the above condition (2) is satisfied requires that

$$
\omega_{01}^{2}=\frac{1}{C_{1}\left(L_{1}+L_{2}\right)}=\omega_{1}^{2}
$$

These considerations result in the condition

$$
\begin{aligned}
& \frac{C_{1}}{C_{0}} \gg \frac{L_{2}}{\left(L_{1}+L_{2}\right)}, \\
\Rightarrow & \frac{C_{1}}{C_{0}} \gg \frac{L_{2}}{L_{1}} \text { for } L_{1} \gg L_{2} .
\end{aligned}
$$

The circuits for $V_{11}$ and $V_{21}$ in Figure 5 a then reduce to the form shown in Figure $5 \mathrm{~b}$. (b)
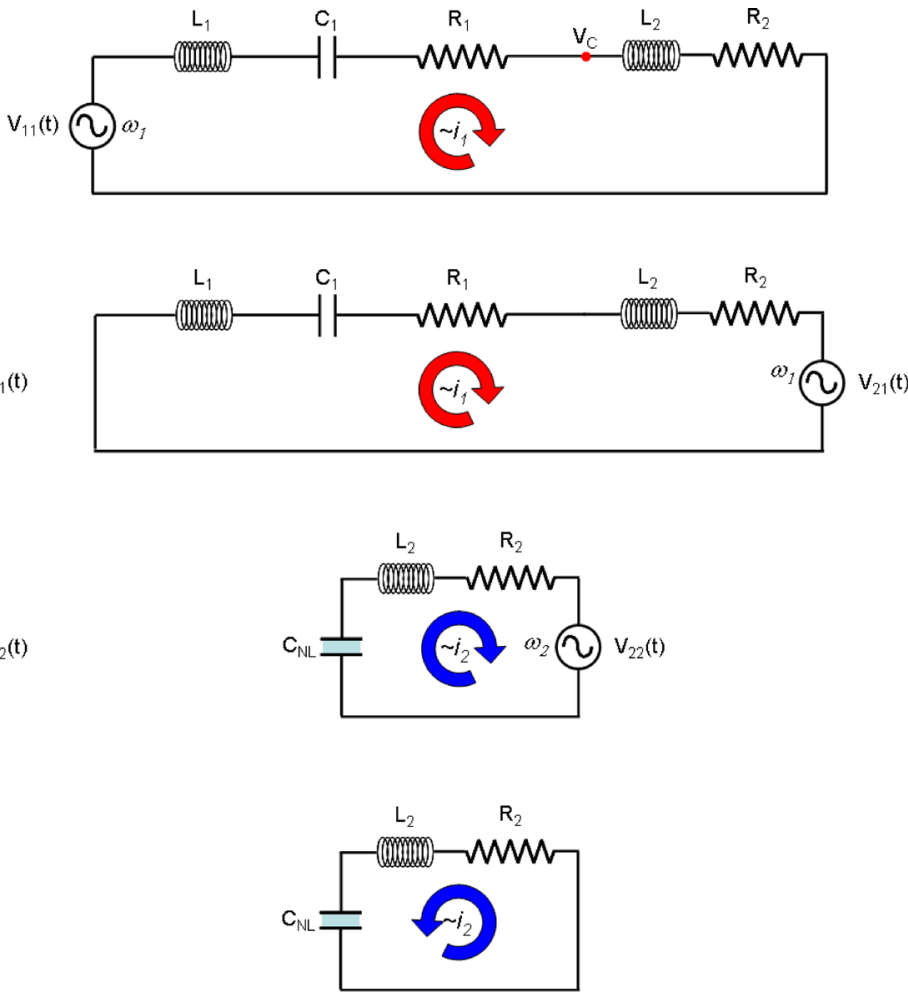

(b) effective circuit seen by each voltage source under the design

It is desirable that the response of the circuit at the controlled beam frequency $\omega_{2}$ is dominated by the effect of $C_{\mathrm{NL}}$, which is set by the controlling beam. To achieve this, at frequency $\omega_{2}$ the impedance of $C_{\mathrm{NL}}$ is made much smaller than $Z_{L_{1} C_{1}}=-\omega_{2} L_{1}+1 /\left(\omega_{2} C_{1}\right)$, the impedance of series combination $L_{1} C_{1}$.

For $\omega_{2}>\omega_{1}$, it follows that $Z_{L_{1} C_{1}}<0$ and $-Z_{L_{1} C_{1}}>0$, and the above requirement can be written as (again taking $\left.C_{N L} \approx C_{0}\right)$

$$
\frac{1}{\omega_{2} C_{0}} \ll \omega_{2} L_{1}-\frac{1}{\omega_{2} C_{1}} \Rightarrow \frac{1}{L_{1}}\left(\frac{1}{C_{0}}+\frac{1}{C_{1}}\right) \ll \omega_{2}^{2} .
$$

Taking $\omega_{2} \approx \omega_{02} \approx\left(L_{2} C_{0}\right)^{-2}$ (noting that $\omega_{02}$ is detuned from but is nevertheless close to $\left.\omega_{2}\right)$ yields

$$
\frac{1}{L_{1}}\left(\frac{1}{C_{0}}+\frac{1}{C_{1}}\right) \ll \frac{1}{L_{2} C_{0}},
$$

which can be written as

$$
\frac{C_{1}}{C_{0}}\left(1-\frac{L_{2}}{L_{1}}\right) \gg \frac{L_{2}}{L_{1}} .
$$

For $L_{1} \gg L_{2}$, the final result is

$$
\frac{C_{1}}{C_{0}} \gg \frac{L_{2}}{L_{1}},
$$

which is the same condition (4b) as that derived earlier in reference to the control beam. Thus the constraints imposed on the circuit by both the control and controlled beams are compatible. Under this condition, the circuits for 
(a)

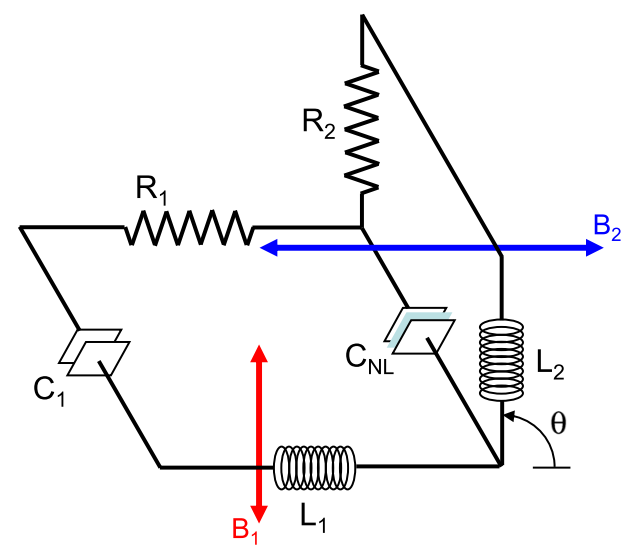

(b)

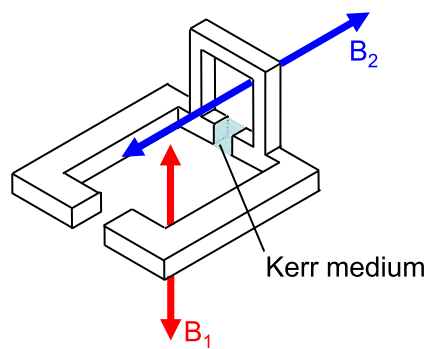

Fig. 6. (a) Folded (3D) dual-resonant conceptual unit cell based on two orthogonally-coupled LCR resonators under two-colour Bfield excitation. The red and blue arrows represent the weak controlling beam and strong controlled beam B-fields, respectively. (b) Dual split-ring resonator conceptual realisation of the generic circuit in (a).

$V_{12}$ and $V_{22}$ in Figure 5a reduce to the form shown in Figure $5 \mathrm{~b}$, and the controlled beam 'sees' a simple LCR circuit with resonance frequency $\omega_{02} \approx\left(L_{2} C_{N L}\right)^{-2}$.

The resonance spectral widths are $\sim\left(R_{1}+R_{2}\right) /\left(L_{1}+L_{2}\right)$ and $\sim R_{2} / L_{2}$ for the metamolecule resonances at $\omega_{01}$ and $\omega_{02}$, respectively. By choosing $R_{1}$ and $R_{2}$ appropriately (either via suitable choice of materials, and by the dimensions of the microstructural features in the metamolecule), the resonance widths can be selected. As discussed above, the resonance at $\omega_{01}$ is made sufficiently broad whilst the resonance at $\omega_{02}$ is made sufficiently narrow (with the exact widths dependent on the requirements demanded by the specific application). The characteristic transient timescale for the system to respond to changes in the intensity of the control beam is $\sim\left(L_{1}+L_{2}\right) /\left(R_{1}+R_{2}\right)$.

Note that in Figure 5 voltage sources $V_{11}$ and $V_{21}$ support each other in terms of producing current flow in both loops, whereas voltage sources $V_{22}$ and $V_{12}$ tend to counteract one another. Whilst this may be useful for limiting the current flow in the circuit due to a strong controlled beam, it also means that the magnetic moments generated in loop 1 and loop 2 oppose each other.

Figure 6a shows a folded 3-dimensional metamolecule unit cell based on two coupled circuit loops under dual Bfield excitation. Taking the fold angle $\theta=90^{\circ}$ and setting the polarisation of the control beam orthogonal to that of the controlled beam, it follows that $V_{12}=V_{21}=0$ and the circuit only oscillates at frequencies $\omega_{1}$ and $\omega_{2}$ in response to $V_{11}$ and $V_{22}$, respectively.

In this case the weak control beam couples to the larger of the two loops (implied by $L_{1}>>L_{2}$ ), whereas the strong controlled beam couples to the smaller of the two loops. This ensures that the metamolecule collects as much of the weak control beam B-field as possible whilst minimising the amount of strong controlled beam B-field collected.

Continuing the analysis with $V_{21}$ and $V_{12}$ set to zero, the peak voltage $V_{C}$ across $C_{\mathrm{NL}}$ in the effective circuit seen by $V_{11}$ in Figure $5 \mathrm{~b}$ is given by

$$
\begin{aligned}
V_{C} \sim\left|V_{11}\right|\left|\frac{-j \omega_{1} L_{2}+R_{2}}{R_{1}+R_{2}}\right| & =\left|V_{11}\right| \cdot \frac{\left(\omega_{1}^{2} L_{2}^{2}+R_{2}^{2}\right)^{1 / 2}}{R_{1}+R_{2}} \\
& =E_{g} d_{g},
\end{aligned}
$$

where $E_{g}$ and $d_{g}$ are the magnitude of the electric field in the capacitor gap and plate separation of the coupling capacitor $C_{\mathrm{NL}}$, respectively. By Faraday's Law, the induced voltage has magnitude $\left|V_{11}\right|=\omega_{1} A_{1} B_{1}$, where $A_{1}$ is the area of loop 1 and $B_{1}$ is the magnitude of the B-field of the controlling beam.

Taking

$$
B_{1}^{2}=\frac{2 \mu_{0} I_{1}}{c},
$$

where $I_{1}$ is the intensity $\left(\mathrm{W} / \mathrm{m}^{2}\right)$ of the control beam and $c$ is the speed of light in vacuum, and combining the above expressions yields

$$
E_{g}^{2}=\frac{2 \omega_{1}^{2} A_{1}^{2} \mu_{0}}{c d_{g}^{2}} \cdot \frac{\left(\omega_{1}^{2} L_{2}^{2}+R_{2}^{2}\right)}{\left(R_{1}+R_{2}\right)^{2}} \cdot I_{1} .
$$

From $C_{N L}=C_{0}+\eta E_{g}^{2}$, it follows that

$$
\begin{aligned}
C_{N L} & =C_{0}+\beta I_{1}, \quad \text { where } \\
\beta & =\eta \cdot \frac{2 \omega_{1}^{2} A_{1}^{2} \mu_{0}}{c d_{g}^{2}} \cdot \frac{\left(\omega_{1}^{2} L_{2}^{2}+R_{2}^{2}\right)}{\left(R_{1}+R_{2}\right)^{2}} .
\end{aligned}
$$

Once the rapid circuit transients have settled down (following a change in $I_{1}$ ), the current $i_{22}$ flowing around loop 2 due to the controlled beam at frequency $\omega_{2}$ is given by

$$
i_{22} \sim \frac{V_{22}}{Z_{2}}=\frac{\omega_{2} A_{2} B_{2} j}{Z_{2}}
$$

where $Z_{2}\left(I_{1}\right)=j\left(-\omega_{2} L_{2}+\frac{1}{\omega_{2} C_{N L}\left(I_{1}\right)}\right)+R_{2}$, 
where $A_{2}$ is the area of loop 2 and $B_{2}$ is the magnitude of the B-field of the controlled beam. The permeability of the metamaterial at frequency $\omega_{2}$, which is determined by the value of $C_{\mathrm{NL}}$ and therefore the intensity of the control beam, is given by $\mu=\mu_{0}\left(1+\mu_{0} m / B_{2}\right)$ where the magnetic moment per unit volume $m=i_{22} A_{2} / V_{m}$ and $V_{m}$ is the volume of the unit cell. Substituting for $i_{22}$ then yields

$$
\mu_{r}\left(I_{1}\right)=\left(1+\frac{j \mu_{0} \omega_{2} A_{2}^{2}}{V_{m} Z_{2}\left(I_{1}\right)}\right), \quad \mu\left(I_{1}\right) \sim \mu_{0} \mu_{r}
$$

where $\mu_{r}$ is the relative permeability. The refractive index $n_{2}$ of the medium at frequency $\omega_{2}$ is then given by $n_{2}\left(I_{1}\right)=\sqrt{\varepsilon_{r} \mu_{r}}$, where $\varepsilon_{r}$ is the relative permittivity.

\subsection{Approximate calculation of $\gamma$}

The parameter $\gamma=d n_{2} / d I_{1}$ (Appendix A) determines the change in the refractive index at frequency $\omega_{2}$ due to a change in the control beam intensity, relative to the $I_{1}=0$ (control beam off) state, and is given by

$$
\frac{d n_{2}}{d I_{1}}=\frac{\varepsilon_{r}^{1 / 2}}{2 \mu_{r}^{1 / 2}} \frac{d \mu_{r}}{d I_{1}} \sim \frac{1}{2} \frac{d \mu_{r}}{d I_{1}}, \quad \varepsilon_{r}=1, \quad \mu_{r} \sim 1 .
$$

Differentiating the relative permeability (Eq. (14)) with respect to $I_{1}$ yields

$$
\frac{d \mu_{r}}{d I_{1}}=-\frac{j \mu_{0} \omega_{2} A_{2}^{2}}{V_{m}} \cdot \frac{1}{Z_{0}^{2}} \cdot \frac{d Z_{2}}{d I_{1}},
$$

where

$$
Z_{0}=Z_{2}\left(I_{1}=0\right)=j\left(-\omega_{2} L_{2}+\frac{1}{\omega_{2} C_{0}}\right)+R_{2},
$$

and

$$
\frac{d Z_{2}}{d I_{1}}=\frac{d Z_{2}}{d C_{N L}} \cdot \frac{d C_{N L}}{d I_{1}}=\frac{d Z_{2}}{d C_{N L}} \cdot \beta .
$$

Defining the detuning parameter $\alpha=\left(\Delta \omega L_{2}\right) / R_{2}$, where $\Delta \omega=\omega_{2}-\omega_{20}$,

$$
\begin{gathered}
\frac{1}{Z_{0}^{2}} \sim \frac{1-4 \alpha^{2}+4 \alpha j}{R^{2}\left(1+4 \alpha^{2}\right)^{2}}, \\
\left.\sim \frac{j-\alpha}{4 R^{2} \alpha^{3}} \text { for } 4 \alpha^{2}>>1 \text { (i.e. } \alpha>>0.5\right),
\end{gathered}
$$

and

$$
\frac{d Z_{2}}{d C_{N L}} \sim \frac{-j}{\omega_{2} C_{0}^{2}}
$$

Combining the expressions above yields the approximate relation

$$
\gamma=\frac{d n_{2}}{d I_{1}} \sim \frac{\mu_{0} A_{2}^{2} \beta}{8 V_{m} \alpha^{2} R_{2}^{2} C_{0}^{2}}-\frac{\mu_{0} A_{2}^{2} \beta}{8 V_{m} \alpha^{3} R_{2}^{2} C_{0}^{2}} j .
$$

The change in the real part of the refractive index $\Delta n_{2 r}$ is therefore proportional to the real part of $\gamma$ :

$$
\Delta n_{2 r}=\gamma_{r} \cdot I_{1}=\frac{\mu_{0} A_{2}^{2} \beta}{8 V_{m} \alpha^{2} R_{2}^{2} C_{0}^{2}} \cdot I_{1},
$$

where

$$
\gamma_{r}=R e(\gamma)=\frac{\mu_{0} A_{2}^{2} \beta}{8 V_{m} \alpha^{2} R_{2}^{2} C_{0}^{2}}
$$

This formula shows that there is a simple relationship between the intensity $I_{1}$ of the control beam and the corresponding refractive index change $\Delta n_{2 r}$ induced in the metamaterial at the controlled beam frequency. Correspondingly, spatiotemporal variations in the intensity $I_{1}$ of the control beam give rise to spatiotemporal variations in the refractive index experienced by the controlled beam, as are required to effect beam zooming and deflection.

\subsection{Circuit realisation and example}

A possible conceptual realisation of the B-field excited system is shown in Figure 6b. This unit cell consists of two split ring resonators arranged orthogonally with respect to each other and sharing a gap containing the nonlinear Kerr medium.

By way of example this system could be employed to effect focussing control of fibre lasers in micromachining applications. For instance, ytterbium ( $\mathrm{Yb}$ ) fibre lasers operating at a wavelength of $1064 \mathrm{~nm}$, repetition rates $>10 \mathrm{kHz}$, pulse duration up to several hundred nanoseconds and average power $>20 \mathrm{~W}$ have been used to machine silicon substrates for use in microelectromechanical systems (MEMS) and the semiconductor industry [44]. In order to control such a laser using the system and method described here, the control laser would preferably be matched to the controlled laser in terms of its repetition rate and pulse duration so that focus control can be utilised during each laser pulse. Examples of potential control lasers include erbium fibre, thulium fibre and holmium: YLF lasers operating at wavelengths of $1550 \mathrm{~nm}, 1900$ $2050 \mathrm{~nm}$ and $2050 \mathrm{~nm}$ or $2060 \mathrm{~nm}$, respectively [45].

For a controlled laser with $a=4 \mathrm{~mm}$ focussed to a spot radius $r_{1}=50 \mu \mathrm{m}$ on a target using a main focussing lens with focal length $f_{2}=250 \mathrm{~mm}$, a weak dynamic lens with $f_{1} \sim 19750 \mathrm{~mm}$ would result in a shift in the focus position of $3.1 \mathrm{~mm}$ to best focus $\left(r_{2}=0 \mu \mathrm{m}\right)$. This assumes the focussed beam behaves according to geometric optics; in reality, the beam is focussed to a finite-sized waist. Taking into account the Gaussian nature of the beam (assuming a typical fibre laser beam quality parameter $M^{2}=1.8$ ), the spot radius on target is reduced from $\sim 63 \mu \mathrm{m}$ to $\sim 38 \mu \mathrm{m}$ when the dynamic lens is activated. For a dynamic lens with length $L=0.2 \mathrm{~mm}$, a refractive index change $\Delta n$ of approximately $2 \times 10^{-3}$ is required. A prototype dual-SRR design is developed below to realise the dynamic lens necessary for this zooming example. 
To design the dual split-ring resonator structure, the dimensions of known single split-ring resonator designs are scaled and then fine-tuned to ensure that the circuit resonance frequencies are correct, according to the operating wavelengths of the control and controlled beams and the prescription given above. For an ytterbium fibre laser operating at a wavelength of $1064 \mathrm{~nm}$ controlled by a Ho:YLF laser operating at $2060 \mathrm{~nm}$, example parameters for the control ring are $l=330 \mathrm{~nm}, w=130 \mathrm{~nm}, t=17 \mathrm{~nm}$ and $d_{q}=90 \mathrm{~nm}$ (for a capacitance plate area of $\left.1.4 \times 10^{-15} \mathrm{~m}^{2}\right)$; example parameters for the controlled ring are $l=100 \mathrm{~nm}, w=50 \mathrm{~nm}, t=15 \mathrm{~nm}$ and $d_{g}=33 \mathrm{~nm}$. Taking $\chi^{(3)} \sim 6 \times 10^{-20}(\mathrm{~m} / \mathrm{V})^{2}$ (e.g. corresponding to potassium dihydrogen phosphate (KDP) crystal or other nonlinear material in the coupling capacitor gap), detuning parameter $\alpha=-3, V_{m}=2 \times 10^{-20} \mathrm{~m}^{3}, \rho=1.59 \times 10^{-8} \Omega \mathrm{m}$ and peak powers of $10 \mathrm{~kW}$ and $1 \mathrm{~kW}$ for the controlled and control beams respectively, the design equation given above yields $\Delta n \sim 2 \times 10^{-3}$, as demanded by the zooming example. For the fibre lasers considered here, a typical pulse duration used for machining is 200 ns and a typical repetition rate is $25 \mathrm{kHz}$. This is a representative example and the parameters can be tuned according to the requirements of the desired application.

It is known that the split ring resonator can be scaled to operate at resonant wavelengths down to $\sim 800 \mathrm{~nm}[21,46]$. At shorter wavelengths, the dual-LCR circuit concept can be retained but a different realisation of the unit cell is likely to be required. Notably, at visible frequencies the flow of displacement current $(J=-i \omega \varepsilon E)$ around the effective circuit dominates that of conduction current $(J=\sigma E)$ which is prevalent at lower frequencies [47]. This section is concluded by offering some conceptual systems which might be developed for use in the visible regime.

For example, at visible frequencies the SRR of Figure 3 can be replaced by a ring of plasmonic nanoparticles around which a circulating displacement current flows in response to a time-varying electric or magnetic field [48]. To realise the dual-SRR circuit of Figure 4, a pair of such rings can be envisaged arranged orthogonally to one another and with at least one nanoparticle with a nonlinear response common to both rings and providing the coupling between them.

Alternatively, the SRR can be replaced by a dielectric particle in a Mie magnetic dipole resonance excited by an electromagnetic field. The displacement current in the particle flows in an effective circuit analogous to that in Figure 3, and LCR parameters can be derived which depend on the size and refractive index of the particle [49]. To realise the dual-LCR circuit of Figure 4, a pair of cojoined dielectric particles can be envisaged, with a nonlinear dielectric region common to both particles providing the coupling between them.

Engheta et al. [50] showed that the lumped-element circuit approach can be extended to the visible regime. Within this 'optical circuit' analogy, the material permittivity takes the role of a conductivity, and circuit connectors (high conductivity) and insulators (low con- ductivity) are correspondingly formed from materials with $|\operatorname{Re}(\varepsilon)|>>\varepsilon_{0}$ and $|\operatorname{Re}(\varepsilon)|<<\varepsilon_{0}$, respectively. Furthermore, 'capacitive' and 'inductive' elements can be formed from materials with $\operatorname{Re}(\varepsilon)>\varepsilon_{0}$ and $\operatorname{Re}(\varepsilon)<\varepsilon_{0}$, respectively, and resistive elements from materials with $\operatorname{Im}(\varepsilon) \neq \varepsilon_{0}$. These concepts might be used to construct a metamaterial with the desired properties operating in the visible regime, and will be the subject of future studies.

\section{Summary}

A system has been described in which the focussing characteristics of a laser beam at one wavelength can be dynamically controlled by a lower-intensity laser beam at another wavelength, via a refracting medium constructed using the principles of metamaterials. By choosing the spatiotemporal intensity characteristics of the control beam, a lens or prism (or more complex optic) can be 'written' into the refracting medium such that the controlled beam is zoomed or deflected, respectively. Such control of the controlled beam potentially leads to large efficiency gains in a range of applications, such as laserdriven inertial confinement fusion.

By placing the refracting medium at the end of an existing beamline the impact on the optical system is minimised, and as the properties of the dynamic optic are set at-a-distance by the control beam, no power supply or other auxiliary components are required in its vicinity. The metamaterial approach allows the response of the refracting medium to be tuned according to the desired operating wavelengths.

A generic metamaterial unit cell, modelled as a dualresonant circuit based on a pair of LCR circuits coupled by a nonlinear capacitor, was proposed and analysed using an approximate approach. The circuit represents a generic metamaterial solution with application across the whole electromagnetic spectrum. Regardless of the frequency of operation, the circuit functions in the same conceptual way: the control beam sets the voltage across the nonlinear coupling capacitor thereby modifying its capacitance; this causes a shift in the second (higher) circuit resonance frequency relative to the frequency of the controlled beam. The response of the circuit at the frequency of the controlled beam, and by extension the effective refractive index of the metamaterial at that frequency, is thereby modified in a controllable way.

Possible circuit realisations were discussed, offering future routes to the construction of a dynamic beam zooming and deflection system operating in the infra-red and visible regions of the electromagnetic spectrum.

Support from the AWE Future Technologies Programme is gratefully acknowledged. The author would also like to thank the referees for their suggestions to improve the manuscript.

(c) British Crown Owned Copyright 2018/AWE applies to this article. 


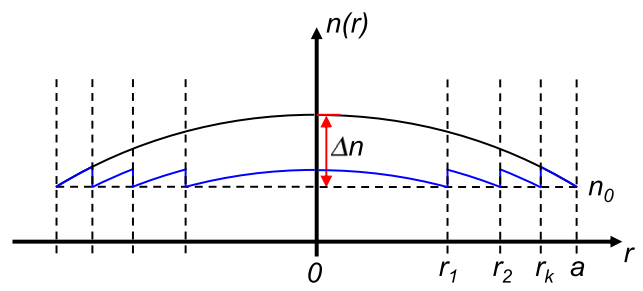

Fig. A1. Spatial refractive index profile for a graded-index positive lens (black curve) and its Fresnel equivalent (blue curve).

\section{Appendix A: Fresnel principle in laser- controlled optics}

\section{A1 Fresnel lens}

As mentioned in Section 2, the Fresnel principle can be applied to laser-controlled optics to reduce the power requirement for the controlling beam. Figure A1 illustrates the spatial refractive index profile for both a graded index lens and its Fresnel equivalent.

For a graded-index lens, the refractive index profile is assumed to be parabolic:

$$
n(r)=n_{0}+\Delta n\left(1-\frac{r^{2}}{a^{2}}\right) .
$$

Here $r$ is the radial spatial coordinate, $a$ is the radius of the lens, $n_{0}$ is the ambient refractive index and $\Delta n$ is the additional refractive index induced on axis by the controlling beam $(\Delta n>0$ for a positive lens). The focal length $f$ of this lens is approximately given by

$$
f \sim \frac{a^{2}}{2 L \Delta n}
$$

where $L$ is the length of the refracting medium.

The induced refractive index $n(r)-n_{0}$ is assumed to be proportional to the controlling beam intensity

$$
n(r)-n_{0}=\gamma \cdot I(r)
$$

where $\gamma$ is the coefficient of proportionality, which in general may be positive or negative depending on the properties of the refracting medium (i.e. in general an increase in the controlling beam intensity may result in an increase or decrease in the refractive index depending on the sign of $\gamma$ ).

The power requirement $P$ for the controlling beam to induce a positive graded-index lens (black curve in Fig. A1) is given by

$$
P=\int_{0}^{a} I(r) \cdot 2 \pi r \cdot d r=\frac{\pi}{2} \cdot \frac{\Delta n}{\gamma} \cdot a^{2} .
$$

For the equivalent Fresnel lens (blue curve in Fig. A1), the spatial intensity profile in the $k$ th segment is given by

$$
I_{k}(r)=I(r)-I\left(r_{k}\right), \quad r_{k-1} \leq r<r_{k} .
$$

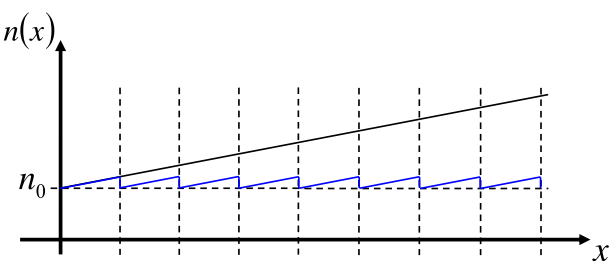

Fig. A2. Spatial refractive index profile for beam deflection (black curve) and Fresnel equivalent (blue curve).

The controlling beam power requirement for the $k$ th segment, $F_{k}$, is then

$$
\begin{aligned}
F_{k} & =\int_{r_{k-1}}^{r_{k}} I_{k}(r) \cdot 2 \pi r \cdot d r \\
& =\int_{r_{k-1}}^{r_{k}} I(r) \cdot 2 \pi r \cdot d r-I\left(r_{k}\right) \int_{r_{k-1}}^{r_{k}} 2 \pi r \cdot d r \\
& =P_{k}-I\left(r_{k}\right) \cdot \pi\left[r_{k}^{2}-r_{k-1}^{2}\right],
\end{aligned}
$$

where $P_{k}=\int_{r_{k-1}}^{r_{k}} I(r) \cdot 2 \pi r \cdot d r$ is the power required to induce a graded refractive index profile in the $k$ th segment. The area of the $k$ th segment $A_{k}=\pi\left[r_{k}^{2}-r_{k-1}^{2}\right]$, hence the expression for $F_{k}$ reduces to

$$
F_{k}=P_{k}-I\left(r_{k}\right) A_{k}
$$

To find the total power required to induce the Fresnel lens $F$, we sum over the power required for each element:

$$
F=\sum_{k=1}^{m} F_{k}=\sum_{k=1}^{m} P_{k}-\sum_{k=1}^{m} I\left(r_{k}\right) A_{k}
$$

where $m$ is the number of Fresnel elements. Taking $P=\sum_{k=1}^{m} P_{k}$ and assuming all segments have equal area $A_{k}=\pi a^{2} / m$ for simplicity,

$$
F=P-A_{k} \sum_{k=1}^{m} I\left(r_{k}\right)=P-\frac{\pi a^{2}}{m} \sum_{j=k}^{m} I\left(r_{k}\right) .
$$

Evaluating the summation on the right-hand side of the above expression,

$$
\begin{aligned}
\sum_{k=1}^{m} I\left(r_{k}\right) & =\frac{\Delta n}{\gamma} \sum_{k=1}^{m}\left(1-\frac{r_{k}^{2}}{a^{2}}\right)=\frac{\Delta n}{\gamma} \sum_{k=1}^{m}\left(1-\frac{k}{m}\right) \\
& =\frac{\Delta n}{\gamma}\left(m-\frac{1}{m} \sum_{k=1}^{m} k\right) \\
& =\frac{\Delta n}{\gamma}\left(m-\frac{(m+1)}{2}\right)=\frac{\Delta n m}{2 \gamma}\left(1-\frac{1}{m}\right) .
\end{aligned}
$$

The expression for $\mathrm{F}$ then reduces to

$$
F=P-\frac{\pi \Delta n a^{2}}{2 \gamma}\left(1-\frac{1}{m}\right)=P-P+\frac{P}{m},
$$


leading to the final result

$$
F=\frac{P}{m}
$$

i.e. that the power requirement to induce a Fresnel lens with $m$ elements of equal area is a factor of $m$ lower than that required to induce a parabolic graded-index lens with the same focal length.

\section{A2 Fresnel Prism}

The same result $F=P / m$ is obtained for a linear refractive index profile (prism), which would be required to implement beam deflection (Fig. A2). A ray passing through a medium of length $L$ and refractive index gradient $d n / \mathrm{d} x$ is deflected by an angle $\sim L \cdot d n / d x$.

\section{References}

1. J. Nuckolls et al., Nature 239, 139 (1972)

2. V.N. Goncharov et al., Plasma Phys. Control. Fusion 59, 014008 (2017)

3. R.S. Craxton et al., Phys. Plas. 22, 110501 (2015)

4. N.B. Meezan et al., Plasma Phys. Control. Fusion 59, 014021 (2017)

5. I.V. Igumenshchev et al., Phys. Rev. Lett. 110, 145001 (2013)

6. D.H. Froula et al., Phys. Plas. 20, 082704 (2013)

7. W. Steen, K.G. Watkins, Y. Mazumder, in: Laser material processing (Springer, London, 2012)

8. R.F. Smith et al., Nature 511, 330 (2014)

9. D.M. Kehne et al., Rev. Sci. Instrum. 84, 013509 (2013)

10. C.A. Haynam et al., Appl. Opt. 46, 3276 (2007)

11. T.R. Boehly et al., Opt. Commun. 133, 495 (1997)

12. J. Ebrardt, J.M. Chaput, J. Phys. Conf. Ser. 112, 032005 (2008)

13. N. Hopps et al., Appl. Opt. 52, 3597 (2013)

14. B. Canaud, F. Garaude, Nucl. Fusion 45, L43 (2005)

15. M. Temporal et al., Phys. Plasmas 17, 022701 (2010)

16. Z. Zhong et al., Opt. Commun. 369, 145 (2016)

17. X. Huang et al., Opt. Express 24, 22051 (2016)
18. I. Golub, Opt. Comm. 94, 143 (1992)

19. M. Rokni, A. Flusberg, IEEE J. Quan. Elec. 20, 1324 (1984)

20. US Patent 4714902 (1987)

21. W. Cai, V. Shalaev, in: Optical metamaterials: fundamentals and applications (Springer, London, 2009)

22. P.J. Wegner et al., in: Proceedings SPIE 5341, Optical engineering at the Lawrence Livermore National Laboratory II: the National Ignition Facility, 2004, 180 p.

23. D.I. Hillier et al., Appl. Opt. 49, 3006 (2010)

24. C. Jeppesen et al., Appl. Phys. Lett. 95, 193108 (2009)

25. N. Katsarakis et al., Appl. Phys. Lett. 84, 2943 (2004)

26. I.V. Shadrivov et al., Opt. Express 20, 9344 (2006)

27. I.V. Shadrivov et al., Opt. Express 16, 20266 (2008)

28. B. Wang et al., Opt. Express 16, 16058 (2008)

29. D.A. Powell et al., Appl. Phys. Lett. 91, 144107 (2007)

30. W.L. Chan et al., Appl. Phys. Lett. 94, 213511 (2009)

31. H.T. Chen et al., Nat. Photon 3, 148 (2009)

32. P.V. Kapitanova et al., Appl. Phys. Lett. 101, 231904 (2012)

33. A.P. Slobozhanyuk et al., JETP Lett. 95, 613 (2012)

34. H.T. Chen et al., Nat. Photon 2, 295 (2008)

35. A. Degiron et al., Opt. Express 15, 1115 (2007)

36. R.W. Boyd, in: Nonlinear optics (Academic Press, London, 2003)

37. J.B. Pendry et al., IEEE Trans. Microwave Theory Tech. 47, 2075 (1999)

38. A.A. Zharov et al., Phys. Rev. Lett. 91, 037401 (2003)

39. A.P. Slobozhanyuk et al., Appl. Phys. Lett. 104, 014104 (2014)

40. P.V. Kapitanova et al., Appl. Phys. Lett. 99, 251914 (2011)

41. J.D. Baena et al., IEEE Trans. Microwave Theory Tech. 53, $1451(2005)$

42. D. Filonov et al., Appl. Phys. Lett. 109, 111904 (2016)

43. E. Poutrina et al., New J. Phys. 12, 093010 (2010)

44. W. O'Neill, K. Li, IEEE J. Sel. Top. Quantum Electron. 15, $462(2009)$

45. V. Ter-Mikirtychev, in: Fundamentals of fiber lasers and fiber amplifiers, Springer series in optical sciences, vol. 181 (Springer, Cham, 2014)

46. M.W. Klein et al., Opt Lett. 31, 1259 (2006)

47. N. Engheta et al., Phys. Rev. Lett. 95, 095504 (2005)

48. A. Alú et al., Opt. Express 14, 1557 (2006)

49. L.Y. Liu et al., Prog. Elec. Res. 116, 159 (2011)

50. N. Engheta et al., Science 317, 1698 (2007)

Cite this article as: Andrew James Comley, Laser beam zooming and deflection using a nonlinear metamaterial refracting medium, EPJ Appl. Metamat. 2018, 5, 8 